\title{
COVID-19 in Singapore - a case series from primary care
}

Sai Zhen $\underline{\mathrm{Sim}}^{1}$, MMed, FCFP, Sok Huang $\underline{\mathrm{TeO}}^{1}$, BSc(Hons), MPH, Jing Wen $\underline{K o n g}^{1}$, MMed, FCFP, Ziliang $\underline{\operatorname{Lim}}^{1}$, MMed, FCFP, Ming Yann Karen $\underline{\mathrm{Ng}}^{1}$, MMed, FCFP, Wern Ee $\underline{\text { Tang }}^{1}$, MMed, FCFP

ABSTRACT As the COVID-19 pandemic worsens, early case detection is vital to limiting community spread. We describe our experiences with four COVID-19 cases at the polyclinics in January and February 2020. This retrospective case series highlights the challenges primary care clinicians face in the early identification of suspect cases based on clinical criteria only. To improve case detection, clinicians can sharpen their clinical acumen by keeping abreast with the latest COVID-19 developments and by maintaining a high state of vigilance.

Keywords: case detection, COVID-19, primary care

\section{INTRODUCTION}

Since the emergence of COVID-19 in Wuhan, China, in December 2019, the disease has spread rapidly worldwide. Singapore was one of the first countries outside China to be affected, with the first imported case on 23 January 2020. ${ }^{(1)}$ On 11 March 2020, the World Health Organization declared COVID-19 a pandemic.

COVID-19 has appeared to be more contagious ${ }^{(2)}$ but less pathogenic than the severe acute respiratory syndrome (SARS) of 2003. (3) The SARS coronavirus 2 (SARS-CoV-2), which causes COVID-19, is thought to be transmitted primarily through large respiratory droplets, although it has also been found in stool and blood. ${ }^{(4)}$ Approximately $10 \%-20 \%$ of people with COVID-19 will require intensive care, ${ }^{(5,6)}$ with a case fatality ratio that has varied widely from $1 \%$ to $7 \% .^{(2,3,6,7)}$ Common risk factors for severe disease outcomes are old age and underlying comorbidities, such as cardiac conditions. ${ }^{(8)}$ Nevertheless, healthy young people and adults are at risk too. ${ }^{(6)}$ It is estimated that one person with COVID-19, even with no or minimal symptoms, may infect two to three others. Hence, early case detection and isolation are important to contain the spread of the virus in the community. ${ }^{(2)}$ However, the symptoms associated with COVID-19 are nonspecific and may share similar clinical and laboratory features as the common cold, influenza and dengue, which is endemic in Singapore. ${ }^{(2,3,5,9)}$ This poses a significant diagnostic challenge to healthcare providers, especially primary care clinicians. ${ }^{(10)}$

In the first two months of the COVID-19 epidemic in Singapore, case detection in primary care was based largely on the suspect case definitions by the Ministry of Health $(\mathrm{MOH})$. Patients who fulfilled the criteria were referred to the hospital for diagnostic testing and further management. The key criteria of a suspect case were the individual's travel history and exposure to confirmed cases. As COVID-19 spreads internationally and locally, the criteria for travel and exposure history have undergone multiple revisions since the start of the outbreak. However, revisions to the case definitions had lagged behind the spread of the virus in the local population.

In this retrospective case series, we reviewed the clinical manifestations of the first four cases of COVID-19 who sought treatment at National Healthcare Group Polyclinics (NHGP) against the prevailing suspect case criteria. Additionally, we discuss the learning points that can contribute to improved case detection and management of COVID-19 in primary care.

This case series was reviewed and approved by the National Healthcare Group Domain Specific Review Board (reference no. 2020/00214), the institutional ethics committee, which granted waiver of informed consent for collection of clinical data from NHGP medical records. Data from NHGP electronic medical records of the four individuals from 1 January 2020 to 29 February 2020 was summarised anonymously by a third party using a standardised data collection form. Information released publicly by $\mathrm{MOH}$ about the cases was also gathered from the $\mathrm{MOH}$ website for reference.

\section{CASE SERIES \\ Patient 1}

Patient 1 was a 39-year-old Singaporean Chinese man, the husband of Patient 2. He worked as a salesperson at a jewellery shop that was frequented by tour groups from China. On 30 January 2020, he presented to a polyclinic with three days of productive cough, runny nose and sore throat, and a fever that had already resolved. Although he did not have any recent travel history to China, he informed the attending doctor that a Chinese tourist who had recently visited his shop had been diagnosed with COVID-19 after returning to China. Patient 1 had not been in direct contact with this tourist; however, he and his wife had been in contact with a colleague who had served the tourist. The colleague had been seen at National Centre for Infectious Diseases (NCID) and told that she did not have COVID-19. 
Table I. Summary of clinical presentations of the four COVID-19 cases seen at the polyclinics.

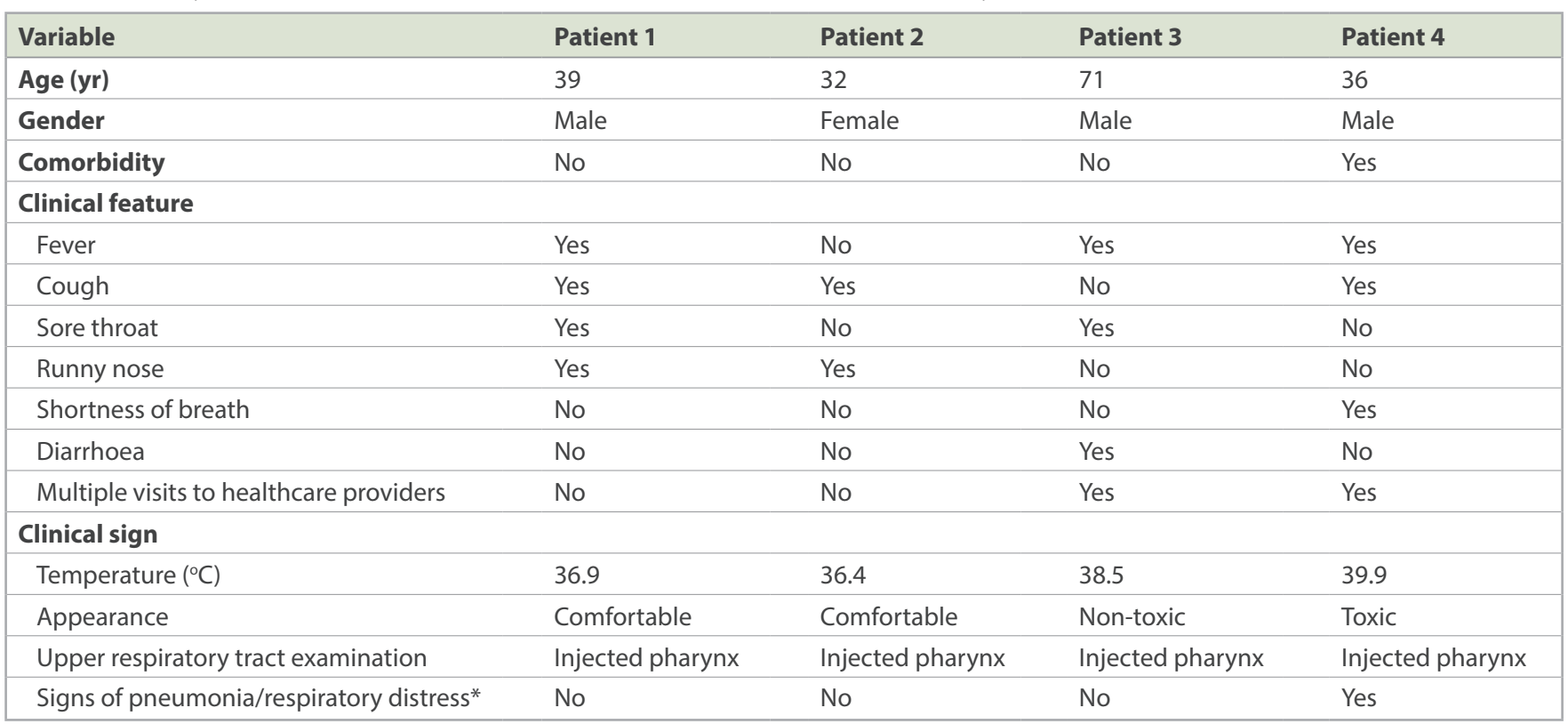

*E.g. crepitations and tachypnoea.

On examination, other than a mildly injected throat, he was afebrile with a body temperature of $36.4^{\circ} \mathrm{C}$ and comfortable at rest. His lungs were clear on auscultation. As he did not fit the COVID-19 suspect case criteria at that time, he was diagnosed with an upper respiratory tract infection (URTI), managed symptomatically, and sent home with the advice to seek medical attention at the emergency department should his condition worsen.

\section{Patient 2}

Patient 2 was a 32-year-old Singaporean Chinese woman, the wife of Patient 1. She worked as a tour guide for tourist groups from China. On 30 January 2020, she also presented to a polyclinic with cough and runny nose of a few days' duration (the exact date of onset was not available). Although she had not travelled out of Singapore recently, she was concerned as both she and her husband had been in close contact with the colleague who had significant interactions with an infected tourist from China. To her knowledge, she had not had contact with a known COVID-19 case and the tourists in her tour group were not from the epicentre areas of Wuhan city or Hubei province.

On examination, other than an injected pharynx, she was comfortable at rest with stable vital signs and a body temperature of $36.9^{\circ} \mathrm{C}$. Her lungs were clear on auscultation. Similar to her husband, Patient 2 did not fit the COVID-19 suspect case criteria at that time. She was diagnosed with URTI, managed symptomatically, and sent home with advice to seek medical care should her symptoms worsen. On 4 February 2020, the polyclinic was informed by the $\mathrm{MOH}$ contact tracing team that both Patients 1 and 2 had been admitted to NCID on 3 February 2020 and had subsequently tested positive for COVID-19. They were discharged from NCID on 12 and 15 February 2020, respectively.

\section{Patient 3}

Patient 3 was a 71-year-old Singaporean Chinese male retiree with pre-existing Type 2 diabetes mellitus, hyperlipidaemia, osteoarthritis and benign prostatic hypertrophy. On 6 February 2020, he presented to the polyclinic with five days of fever and sore throat, and two episodes of loose stools two days prior. He had seen a general practitioner (GP) on 5 February 2020 and had been given medications for his symptoms. However, his fever persisted. He had not travelled out of Singapore recently and had no close contact with a known COVID-19 case.

Physical examination at the polyclinic revealed a body temperature of $38.5^{\circ} \mathrm{C}$ and a mildly injected pharynx. There were no signs of pneumonia or respiratory distress. The patient had already had blood tests at the GP clinic on 5 February 2020 and was keen to follow up on his test results with the GP. As he did not fulfil the prevailing $\mathrm{MOH}$ suspect case criteria and diagnostic testing was not available in primary care then, he was diagnosed with influenza-like illness and managed symptomatically. He was sent home with advice to follow up with his GP. On 8 February 2020 , the clinic was informed by the $\mathrm{MOH}$ contact tracing team that Patient 3 had been admitted to NCID on 7 February 2020 for pneumonia and had subsequently tested positive for COVID-19. He was still warded in NCID at the time of this report.

\section{Patient 4}

Patient 4 was a 36-year-old male Chinese national who worked as a food courier. He presented to the clinic on the afternoon of 19 February 2020 and reported having fever, productive cough and sore throat since 14 February 2020. He had visited another GP clinic three times on 14 February, 16 February and the morning of 19 February 2020, and been prescribed antibiotics (he was unable to name the antibiotics) for his condition on 14 February 2020. However, his condition worsened on 19 February 2020 with the development of breathlessness. He had not travelled out 
of Singapore recently and had no close contact with a known COVID-19 case.

On examination, he was febrile with a body temperature of $39.9^{\circ} \mathrm{C}$, blood pressure of $108 / 70 \mathrm{mmHg}$, pulse rate of 147 beats per minute, respiratory rate of 26 breaths per minute, and oxygen saturation of $86 \%$ on room air. His pharynx was injected and on auscultation, air entry was reduced over the right upper lobe of his lungs. He was diagnosed with pneumonia, given supplemental oxygen and an intravenous normal saline drip, and conveyed by ambulance to Khoo Teck Puat Hospital, where he was admitted. On 20 February 2020, the clinic was informed that Patient 4 had tested positive for COVID-19 and that he had been intubated and sent to NCID for further management. He was discharged from NCID on 7 March 2020

Table I summarises the clinical presentations of the four cases who visited the polyclinic. Table II compares the prevailing suspect case criteria against the time that each case sought their consultation at the polyclinic.

\section{DISCUSSION}

This retrospective case series reports the clinical features of four COVID-19 patients who visited the polyclinics in the first two months of the epidemic in Singapore, vis-à-vis the prevailing suspect case criteria at the time of their presentation at the polyclinic.

These four cases highlight the challenges that primary healthcare providers face in identifying patients with COVID-19. COVID-19 symptoms can range from brief, nonspecific and mild to more severe, regardless of whether the patients had travelled to affected places or had been exposed to known cases. Our findings are similar to other case reports from Germany, ${ }^{(11)}$ Taiwan, ${ }^{(12)}$ Thailand ${ }^{(13)}$ and the United States. ${ }^{(14)}$

Even with a sensitive surveillance system, patients with mild COVID-19 will still be missed. ${ }^{(15)}$ Patients 1 and 2 were among the first few cases of local transmission of COVID-19 in Singapore. They had mild symptoms and did not fulfil the prevailing $\mathrm{MOH}$ suspect case criteria, which focused on travel to China. As the revisions to the case definitions lagged behind the spread of the virus in the local population, there was a delay in the identification of some of these early cases.

Based on these cases, a few observations and lessons drawn may aid the future detection of COVID-19 in primary care. Firstly, it is essential to obtain detailed information on the patients' symptoms, travel history and social interactions for possible sources of contact with COVID-19. Secondly, persistent or worsening respiratory symptoms and multiple presentations at other clinics are red flags that warrant closer assessment. Thirdly, longitudinal observation can help differentiate COVID-19 from other illnesses. As emphasised by McWhinney, ${ }^{(16)}$ time can be used as a diagnostic tool, and clinicians should communicate clearly to patients about self-monitoring and the need for followup with the same clinician for continuity of care. Lastly, in addition to keeping abreast of the latest $\mathrm{MOH}$ case definitions, clinicians need to keep updated on developments in the COVID-19 outbreak and SARS-CoV-2 biology and epidemiology, to help them identify high-risk patients.

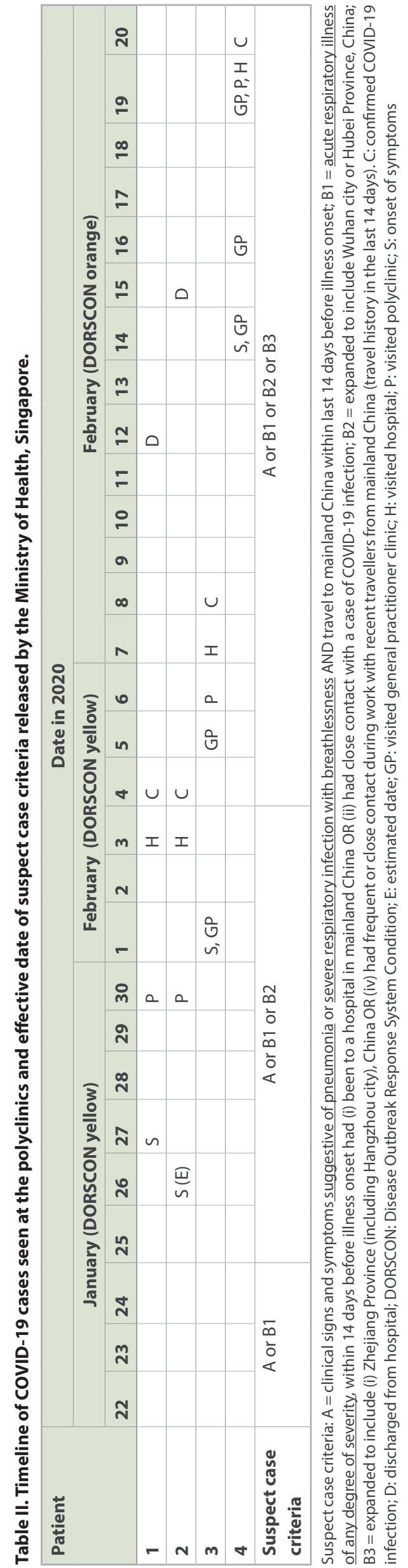


Early detection of COVID-19 cases is crucial to containing the outbreak in Singapore. However, as illustrated by the four cases, primary care clinicians face significant challenges in identifying patients at risk of the infection. The nonspecific presentation of COVID-19, its rapid progress, and the need to concurrently manage the disease while trying to understand its nature place huge demands on frontline healthcare workers. While we await the availability of effective point-of-care testing, clinicians can sharpen their clinical acumen by keeping abreast of the latest COVID-19 developments and maintaining a high state of vigilance to improve early COVID-19 case detection.

\section{ACKNOWLEDGEMENTS}

The authors would like to thank colleagues from Hougang Polyclinic and Yishun Polyclinic, as well as Ms Nisha Menon, Nursing Services, NHGP, for their assistance in the preparation of the manuscript. This research is supported by the Ministry of Health, Singapore, National Medical Research Council under the Centre Grant Programme (ref no.: NMRC/CG/C019/2017).

\section{REFERENCES}

1. Lum LHW, Tambyah PA. Outbreak of COVID-19 - an urgent need for good science to silence our fears? Singapore Med J 2020; 61:55-7.

2. Del Rio C, Malani PN. COVID-19-new insights on a rapidly changing epidemic.
JAMA 2020; 323:1339-40.

3. Guan WJ, Ni ZY, Hu Y, et al. Clinical characteristics of coronavirus disease 2019 in China. N Engl J Med 2020; 382:1708-20.

4. U.S. Centers for Disease Control and Prevention. How COVID-19 Spreads, Available at: https://www.cdc.gov/coronavirus/2019-ncov/about/transmission. html. Accessed Mar, 2020.

5. Young BE, Ong SWX, Kalimuddin S, et al. Epidemiologic features and clinical course of patients infected with SARS-CoV-2 in Singapore. JAMA 2020; 323:1488-94.

6. CDC COVID-19 Response Team. Severe outcomes among patients with coronavirus disease 2019 (COVID-19) - United States, February 12-March 16, 2020. MMWR Morb Mortal Wkly Rep 2020; 69:343-6.

7. Onder G, Rezza G, Brusaferro S. Case-fatality rate and characteristics of patients dying in relation to COVID-19 in Italy. JAMA 2020; 323:1775-6.

8. Cherian R, Poh KK. At the 'heart' of the COVID-19 outbreak: early cardiac implications and mitigating strategies. Singapore Med J 2020; 61:373-4.

9. Yan G, Lee CK, Lam LTM, et al. Covert COVID-19 and false-positive dengue serology in Singapore. Lancet Infect Dis 2020; 20:536.

10. Wong JEL, Leo YS, Tan CC. COVID-19 in Singapore-current experience: critical global issues that require attention and action. JAMA 2020; 323:1243-4.

11. Rothe C, Schunk M, Sothmann P, et al. Transmission of 2019-nCoV infection from an asymptomatic contact in Germany. N Engl J Med 2020; 382:970-1.

12. Liu YC, Liao CH, Chang CF, Chou CC, Lin YR. A locally transmitted case of SARS-CoV-2 infection in Taiwan. N Engl J Med 2020; 382:1070-2.

13. Pongpirul WA, Pongpirul K, Ratnarathon AC, Prasithsirikul W. Journey of a Thai taxi driver and novel coronavirus. N Engl J Med 2020; 382:1067-8.

14. Holshue ML, DeBolt C, Lindquist S, et al. First case of 2019 novel coronavirus in the United States. N Engl J Med 2020; 382:929-36.

15. Wilder-Smith A, Chiew CJ, Lee VJ. Can we contain the COVID-19 outbreak with the same measures as for SARS? Lancet Infect Dis 2020; 20:e102-7.

16. McWhinney IR. A Textbook of Family Medicine. 2nd ed. New York: Oxford University Press, 1997. 